\title{
Multi-Team Bertrand Game with Heterogeneous Players
}

\author{
Mohammed Fathy Elettreby ${ }^{{ }^{*}}$, Daoud Suleiman Mashat ${ }^{2}$, Ashraf Mobarez Zenkour ${ }^{2}$ \\ ${ }^{1}$ Mathematics Department, Faculty of Science, King Khaled University, Abha, Saudi Arabia \\ ${ }^{2}$ Department of Mathematics, Faculty of Science, King Abdulaziz University, Jeddah, Saudi Arabia \\ E-mail: "mohfathy@mans.edu.eg \\ Received March 9, 2011; revised June 8, 2011; accepted June 15, 2011
}

\begin{abstract}
In this paper, we proposed a general form of a multi-team Bertrand game. Then, we studied a two-team Bertrand game, each team consists of two firms, with heterogeneous strategies among teams and homogeneous strategies among players. We find the equilibrium solutions and the conditions of their local stability. Numerical simulations were used to illustrate the complex behaviour of the proposed model, such as period doubling bifurcation and chaos. Finally, we used the feedback control method to control the model.
\end{abstract}

Keywords: Bertrand Game, Non-Convex Dynamical Multi-Team Game, Incomplete Information Dynamical System, Marginal Profit Method, Nash Equilibrium

\section{Introduction}

Game theory $[1,2]$ is the study of multi-person decision problem. Such problems arise in economics. The game is called incomplete information if at least one of the players does not know the other player's payoff, such as in an auction when the bidders do not know the offers of each other. Otherwise, it is called complete information game. Also, the game can be classified to static or dynamic game. There are two famous economic games, the first is the Cournot game [3] and the second is the Bertrand game [4]. In economic games, the first step is to construct the game. The second step is to solve the game (get their Nash equilibrium) and study the stability of these equilibria. Nash [5] showed that in any finite game there exists at least one Nash equilibrium.

Nature push us to make teams in all fields. This has at least two main advantages. The first is the improvement of our profit and the second is that living in a team reduces the risk. For example, in the forest animals live in teams (herds). Since looking for food in a team is more efficient than doing it alone and reduces predation risk due to early spotting of predators and that existing in a team gives a higher probability that the predator will attack another member of the team. Another example is the competition between firms in the market. Suppose $M$ branches of McDonald fast food shops compete against $N$ branches of Kentucky fast food shops.

Multi-team game has been studied in [6]. In their work, they proposed and applied the concept of multi-team game in the hock-dove game, prisoner dilemma game and Cournot game. Also, the Cournot multi-team game has been studied in [7-11]. The standard static Bertrand game has been studied in [12]. A duopoly Bertrand game with bounded rationality is studied in [13]. Multi-team bertrand game is studied in [14] with two teams but the second team consists of one player.

We will construct the model in Section 2. In Section 3, we will analysis the model, i.e., we will find its equilibrium points and their stability conditions. Some numerical analysis will be done in Section 4 to show the complexity behaviour of the model. Finally in section 5, we will use the feedback control method to control our model.

\section{The Model}

Bertrand game is a model of competition used in economics. It describes interaction among firms that set prices and their customers that choose quantities at that price. In this game there are at least two firms producing homogeneous products and compete by setting prices simultaneously. Consumers buy everything from a firm with a lower price. If all firms have the same price, consumers randomly select among them.

Suppose there are totally $n$ firms (produce certain product) in the market and these firms are divided into $N$ teams. Let $p_{i j}$ be the price per unit of that product produced by the firm $j$ in the team $i$ and let $c_{i j}$ be the marginal cost of producing one unit of that product 
by the firm $j$ in the team $i$. Then, the payoff of the firm $j$ in the team $i$, if it played without the team, is given by the following equations;

$$
\begin{aligned}
& \pi_{i j}=\left(p_{i j}-c_{i j}\right)\left(a-b p_{i j}+\Sigma p_{l k}-p_{i j}\right), \\
& i=1, \cdots, N, j=1, \cdots, N_{i},
\end{aligned}
$$

where $N$ is the number of teams and $N_{i}$ is the number of firms in the team $i$. The positive constants $a, b$ are the demand parameters where $b$ is the slop of the demand function.

We propose that, firms in the same team share some of their payoffs with their team mates. So, let $\varepsilon_{l j}^{i}$ be the payoff rate that firm $j$ will takes from the payoff of firm $l$ in the same team $i$. It is clear that $0<\varepsilon_{l j}^{i}<1$, $\sum_{l} \varepsilon_{l j}^{i}<1$ and $\sum_{j} \varepsilon_{l j}^{i}<1$. For example, the final payoff of the first firm $(j=1)$ of the first team $(i=1)$, if he played with the team, is given by the followimg;

$$
\begin{aligned}
\Pi_{11}= & \left(1-\varepsilon_{12}^{1}-\varepsilon_{13}^{1}-\cdots-\varepsilon_{1 N_{1}}^{1}\right) \pi_{11} \\
& +\varepsilon_{21}^{1} \pi_{12}+\varepsilon_{31}^{1} \pi_{13}+\cdots+\varepsilon_{N_{1} 1}^{1} \pi_{1 N_{1}},
\end{aligned}
$$

where $N_{1}$ is the number of firms in the first team. In general, the final payoff of the firm $j$ in the team $i$ is given by;

$$
\Pi_{i j}=\left(1-\sum_{l \neq j}^{N_{i}} \varepsilon_{j l}^{i}\right) \pi_{i j}+\sum_{l \neq j}^{N_{i}} \varepsilon_{l j}^{i} \pi_{i l}
$$

In the case of two teams $(N=2)$ where each team consists of two firms $\left(N_{1}=N_{2}=2\right)$ and from Equation (1), we get the following payoffs of the firms in each team;

$$
\begin{aligned}
& \pi_{11}=\left(p_{11}-c_{11}\right)\left(a-b p_{11}+p_{12}+p_{21}+p_{22}\right), \\
& \pi_{12}=\left(p_{12}-c_{12}\right)\left(a-b p_{12}+p_{11}+p_{21}+p_{22}\right), \\
& \pi_{21}=\left(p_{21}-c_{21}\right)\left(a-b p_{21}+p_{22}+p_{11}+p_{12}\right), \\
& \pi_{22}=\left(p_{22}-c_{22}\right)\left(a-b p_{22}+p_{21}+p_{11}+p_{12}\right) .
\end{aligned}
$$

Using the assumption in Equation (2) of sharing some of the payoffs and Equation (3), we get the final payoffs of the firms as follows:

$$
\begin{aligned}
& \Pi_{11}=\left(1-\varepsilon_{12}^{1}\right) \pi_{11}+\varepsilon_{21}^{1} \pi_{12}, \\
& \Pi_{12}=\left(1-\varepsilon_{21}^{1}\right) \pi_{12}+\varepsilon_{12}^{1} \pi_{11}, \\
& \Pi_{21}=\left(1-\varepsilon_{12}^{2}\right) \pi_{21}+\varepsilon_{21}^{2} \pi_{22}, \\
& \Pi_{22}=\left(1-\varepsilon_{21}^{2}\right) \pi_{22}+\varepsilon_{12}^{2} \pi_{21} .
\end{aligned}
$$

In this model, we assume that the firms in the first team use the marginal profit method [15], to expect their profit for the next time according to the following equations;

$$
p_{1 j}^{t+1}=p_{1 j}^{t}+\alpha_{1 j}\left(p_{1 j}^{t}\right) \frac{\partial \Pi_{1 j}^{t}}{\partial p_{1 j}^{t}}, j=1,2,
$$

where $\alpha_{1 j}$ is the speed (rate) of adjustment and it is a function of the price $p_{1 j}$. The firms in the second team use Nash equilibrium [2], to make their decision for the next step by solving the following equations;

$$
\frac{\partial \Pi_{2 j}^{t}}{\partial p_{2 j}^{t}}=0, j=1,2 .
$$

In this model, we assume that the speed of adjustment will be linear and take the form $\alpha_{1 j}\left(p_{1 j}^{t}\right)=\alpha_{1 j} p_{1 j}^{t}$, and $\alpha_{1 j}>0$. Substituting from Equation (4) in Equations (5) and (6), we get the following system (7);

Then, Equation (7) describe a system of two teams, each team consists of two firms with homogeneous strategies among each firms in each team and heterogeneous strategies among teams.

\section{The Analysis of the Model}

The steady state (equilibrium) solutions are very interest [16]. In the context of difference equations, an equilibrium solution $x$ is defined to be the value that satisfies the relations $x_{t+1}=x_{t}=x$. Then, we can get the equilibrium solutions for our model by the following. Let

$$
\begin{aligned}
& p_{11}^{t+1}=p_{11}^{t}+\alpha_{11} p_{11}^{t}\left[\left(1-\varepsilon_{12}^{1}\right)\left(a+b c_{11}-2 b p_{11}^{t}+p_{12}^{t}+p_{21}^{t}+p_{22}^{t}\right)+\varepsilon_{21}^{1}\left(p_{12}^{t}-c_{12}\right)\right], \\
& p_{12}^{t+1}=p_{12}^{t}+\alpha_{12} p_{12}^{t}\left[\left(1-\varepsilon_{21}^{1}\right)\left(a+b c_{12}-2 b p_{12}^{t}+p_{11}^{t}+p_{21}^{t}+p_{22}^{t}\right)+\varepsilon_{12}^{1}\left(p_{11}^{t}-c_{11}\right)\right], \\
& p_{21}^{t+1}=\frac{1}{2 b}\left(a+b c_{21}+p_{22}^{t}+p_{11}^{t}+p_{12}^{t}\right)+\frac{\varepsilon_{21}^{2}}{2 b\left(1-\varepsilon_{12}^{2}\right)}\left(p_{22}^{t}-c_{22}\right), \\
& p_{22}^{t+1}=\frac{1}{2 b}\left(a+b c_{22}+p_{21}^{t}+p_{11}^{t}+p_{12}^{t}\right)+\frac{\varepsilon_{12}^{2}}{2 b\left(1-\varepsilon_{21}^{2}\right)}\left(p_{21}^{t}-c_{21}\right) .
\end{aligned}
$$




$$
\begin{aligned}
& \begin{array}{l}
p_{11}^{t+1}=p_{11}^{t}=p_{11}, p_{12}^{t+1}=p_{12}^{t}=p_{12}, \\
p_{21}^{t+1}=p_{21}^{t}=p_{21}, p_{22}^{t+1}=p_{22}^{t}=p_{22} .
\end{array} \\
& \alpha_{11} p_{11}^{t}\left[\left(1-\varepsilon_{12}^{1}\right)\left(a+b c_{11}-2 b p_{11}+p_{12}+p_{21}+p_{22}\right)+\varepsilon_{21}^{1}\left(p_{12}-c_{12}\right)\right]=0, \\
& \alpha_{12} p_{12}^{t}\left[\left(1-\varepsilon_{21}^{1}\right)\left(a+b c_{12}-2 b p_{12}+p_{11}+p_{21}+p_{22}\right)+\varepsilon_{12}^{1}\left(p_{11}-c_{11}\right)\right]=0, \\
& p_{21}=\frac{1}{2 b}\left(a+b c_{21}+p_{22}+p_{11}+p_{12}\right)+\frac{\varepsilon_{21}^{2}}{2 b\left(1-\varepsilon_{12}^{2}\right)}\left(p_{22}-c_{22}\right), \\
& p_{22}=\frac{1}{2 b}\left(a+b c_{22}+p_{21}+p_{11}+p_{12}\right)+\frac{\varepsilon_{12}^{2}}{2 b\left(1-\varepsilon_{21}^{2}\right)}\left(p_{21}-c_{21}\right) .
\end{aligned}
$$

Then, using Equation (8), the equilibrium points are

We get three boundary equilibrium solution points and the fourth one is the coexistence equilibrium one. The first boundary equilibrium one is given by

$$
E_{1}=\left(0,0, \frac{l_{1}}{d_{1}}, \frac{l_{2}}{d_{1}}\right), \text { where }
$$

$$
\begin{gathered}
d_{1}=4 b^{2}\left(1-\varepsilon_{12}^{2}\right)\left(1-\varepsilon_{21}^{2}\right)-\left(1-\varepsilon_{21}^{2}+\varepsilon_{12}^{2}\right)\left(1-\varepsilon_{12}^{2}+\varepsilon_{21}^{2}\right), \\
l_{1}=a\left(1-\varepsilon_{21}^{2}\right)\left(2 b\left(1-\varepsilon_{12}^{2}\right)+\left(1-\varepsilon_{12}^{2}+\varepsilon_{21}^{2}\right)\right)+c_{21}\left(2 b^{2}\left(1-\varepsilon_{12}^{2}\right)\left(1-\varepsilon_{21}^{2}\right)-\varepsilon_{12}^{2}\left(1-\varepsilon_{12}^{2}+\varepsilon_{21}^{2}\right)\right)+c_{22} b\left(1-\varepsilon_{21}^{2}\right)\left(1-\varepsilon_{12}^{2}-\varepsilon_{21}^{2}\right), \\
l_{2}=a\left(1-\varepsilon_{12}^{2}\right)\left(2 b\left(1-\varepsilon_{21}^{2}\right)+\left(1-\varepsilon_{21}^{2}+\varepsilon_{12}^{2}\right)\right)+c_{21} b\left(1-\varepsilon_{12}^{2}\right)\left(1-\varepsilon_{21}^{2}-\varepsilon_{12}^{2}\right)+c_{22}\left(2 b^{2}\left(1-\varepsilon_{21}^{2}\right)\left(1-\varepsilon_{12}^{2}\right)-\varepsilon_{21}^{2}\left(1-\varepsilon_{21}^{2}+\varepsilon_{12}^{2}\right)\right) .
\end{gathered}
$$

The second boundary equilibrium one is given by $E_{2}=\left(0, p_{12}, \frac{n_{1}}{d_{2}}, \frac{n_{2}}{d_{2}}\right)$, where

$$
\begin{aligned}
d_{2}=\left(1-\varepsilon_{21}^{1}\right) & \left(\left(4 b^{2}-1\right)^{2}\left(1-\varepsilon_{12}^{2}\right)\left(1-\varepsilon_{21}^{2}\right)-\left((1+2 b)\left(1-\varepsilon_{12}^{2}\right)+2 b \varepsilon_{21}^{2}\right)\left((1+2 b)\left(1-\varepsilon_{21}^{2}\right)+2 b \varepsilon_{12}^{2}\right)\right), \\
n_{1}= & 2 b\left(1-\varepsilon_{21}^{2}\right)\left((1+2 b)\left(1-\varepsilon_{12}^{2}\right)+\varepsilon_{21}^{2}\right)\left(a(1+2 b)\left(1-\varepsilon_{21}^{1}\right)-\varepsilon_{12}^{1} c_{11}+b\left(1-\varepsilon_{21}^{1}\right) c_{12}\right) \\
& +2 b\left(1-\varepsilon_{21}^{1}\right) c_{21}\left(b\left(4 b^{2}-1\right)\left(1-\varepsilon_{12}^{2}\right)\left(1-\varepsilon_{21}^{2}\right)-\varepsilon_{12}^{2}\left((1+2 b)\left(1-\varepsilon_{12}^{2}\right)+2 b \varepsilon_{21}^{2}\right)\right) \\
& +2 b\left(1-\varepsilon_{21}^{1}\right)\left(1-\varepsilon_{21}^{2}\right) c_{22}\left(-\varepsilon_{21}^{2}\left(4 b^{2}-1\right)+b\left((1+2 b)\left(1-\varepsilon_{12}^{2}\right)+2 b \varepsilon_{21}^{2}\right)\right), \\
n_{2}= & 2 b\left(1-\varepsilon_{12}^{2}\right)\left((1+2 b)\left(1-\varepsilon_{21}^{2}\right)+\varepsilon_{12}^{2}\right)\left(a(1+2 b)\left(1-\varepsilon_{21}^{1}\right)-\varepsilon_{12}^{1} c_{11}+b\left(1-\varepsilon_{21}^{1}\right) c_{12}\right) \\
& +2 b\left(1-\varepsilon_{21}^{1}\right)\left(1-\varepsilon_{12}^{2}\right) c_{21}\left(-\varepsilon_{12}^{2}\left(4 b^{2}-1\right)+b((1+2 b))\left(1-\varepsilon_{21}^{2}\right)+2 b \varepsilon_{12}^{2}\right) \\
& +2 b\left(1-\varepsilon_{21}^{1}\right) c_{22}\left(b\left(4 b^{2}-1\right)\left(1-\varepsilon_{12}^{2}\right)\left(1-\varepsilon_{21}^{2}\right)-\varepsilon_{21}^{2}\left((1+2 b)\left(1-\varepsilon_{21}^{2}\right)+2 b \varepsilon_{12}^{2}\right)\right) .
\end{aligned}
$$

and

$$
\begin{aligned}
& \text { and } \\
& \begin{aligned}
& p_{12}=\frac{\left(a+b c_{12}\right) d_{2}+n_{1}+n_{2}}{2 b d_{2}}-\frac{\varepsilon_{12}^{1} c_{11}}{2 b\left(1-\varepsilon_{21}^{1}\right)} . \text { The third boundary equilibrium one is given by } \\
& d_{3}=\left(1-\varepsilon_{12}^{1}\right)\left(\left(4 b^{2}-1\right)^{2}\left(1-\varepsilon_{12}^{2}\right)\left(1-\varepsilon_{21}^{2}\right)-\left((1+2 b)\left(1-\varepsilon_{12}^{2}\right)+2 b \varepsilon_{21}^{2}\right)\left((1+2 b)\left(1-\varepsilon_{21}^{2}\right)+2 b \varepsilon_{12}^{2}\right)\right) \\
& m_{1}=2 b\left(1-\varepsilon_{21}^{2}\right)\left((1+2 b)\left(1-\varepsilon_{12}^{2}\right)+\varepsilon_{21}^{2}\right)\left(a(1+2 b)\left(1-\varepsilon_{12}^{1}\right)+b\left(1-\varepsilon_{12}^{1}\right) c_{11}-\varepsilon_{21}^{1} c_{12}\right) \\
&+ \\
&+2 b\left(1-\varepsilon_{12}^{1}\right) c_{21}\left(b\left(4 b^{2}-1\right)\left(1-\varepsilon_{12}^{2}\right)\left(1-\varepsilon_{21}^{2}\right)-\varepsilon_{12}^{2}\left((1+2 b)\left(1-\varepsilon_{12}^{2}\right)+2 b \varepsilon_{21}^{2}\right)\right) \\
&+ 2 b\left(1-\varepsilon_{12}^{1}\right)\left(1-\varepsilon_{21}^{2}\right) c_{22}\left(-\varepsilon_{21}^{2}\left(4 b^{2}-1\right)+b\left((1+2 b)\left(1-\varepsilon_{12}^{2}\right)+2 b \varepsilon_{21}^{2}\right)\right),
\end{aligned}
\end{aligned}
$$




$$
\begin{aligned}
m_{2} & =2 b\left(1-\varepsilon_{12}^{2}\right)\left((1+2 b)\left(1-\varepsilon_{21}^{2}\right)+\varepsilon_{12}^{2}\right)\left(a(1+2 b)\left(1-\varepsilon_{12}^{1}\right)+b\left(1-\varepsilon_{12}^{1}\right) c_{11}-\varepsilon_{21}^{1} c_{12}\right) \\
& +2 b\left(1-\varepsilon_{12}^{1}\right)\left(1-\varepsilon_{12}^{2}\right) c_{21}\left(-\varepsilon_{12}^{2}\left(4 b^{2}-1\right)+b\left((1+2 b)\left(1-\varepsilon_{21}^{2}\right)+2 b \varepsilon_{12}^{2}\right)\right) \\
& +2 b\left(1-\varepsilon_{12}^{1}\right) c_{22}\left(b\left(4 b^{2}-1\right)\left(1-\varepsilon_{12}^{2}\right)\left(1-\varepsilon_{21}^{2}\right)-\varepsilon_{21}^{2}\left((1+2 b)\left(1-\varepsilon_{21}^{2}\right)+2 b \varepsilon_{12}^{2}\right)\right) .
\end{aligned}
$$

and

$$
\begin{gathered}
p_{11}=\frac{\left(a+b c_{11}\right) d_{3}+m_{1}+m_{2}}{2 b d_{3}}-\frac{\varepsilon_{21}^{1} c_{12}}{2 b\left(1-\varepsilon_{12}^{1}\right)} . \quad E_{4}=\left(p_{11}^{4}, p_{12}^{4}, p_{21}^{4}, p_{22}^{4}\right), \text { where } \\
p_{11}^{4}=\frac{2 a\left(B_{4}-B_{3}\right)+c_{11}\left(D_{3} B_{4}-F_{4} B_{3}\right)+c_{12}\left(E_{3} B_{4}-H_{4} B_{3}\right)+c_{21}\left(F_{3} B_{4}-D_{4} B_{3}\right)+c_{22}\left(H_{3} B_{4}-E_{4} B_{3}\right)}{A_{4} B_{3}-A_{3} B_{4}}, \\
p_{12}^{4}=\frac{2 a\left(B_{2}-B_{1}\right)+c_{11}\left(D_{1} B_{2}-F_{2} B_{1}\right)+c_{12}\left(E_{1} B_{2}-H_{2} B_{1}\right)+c_{21}\left(F_{1} B_{2}-D_{2} B_{1}\right)+c_{22}\left(H_{1} B_{2}-E_{2} B_{1}\right)}{A_{2} B_{1}-A_{1} B_{2}}, \\
p_{21}^{4}=\frac{2 a\left(A_{4}-A_{3}\right)+c_{11}\left(D_{3} A_{4}-F_{4} A_{3}\right)+c_{12}\left(E_{3} A_{4}-H_{4} A_{3}\right)+c_{21}\left(F_{3} A_{4}-D_{4} A_{3}\right)+c_{22}\left(H_{3} A_{4}-E_{4} A_{3}\right)}{B_{4} A_{3}-B_{3} A_{4}}, \\
p_{12}^{4}=\frac{2 a\left(A_{2}-A_{1}\right)+c_{11}\left(D_{1} A_{2}-F_{2} A_{1}\right)+c_{12}\left(E_{1} A_{2}-H_{2} A_{1}\right)+c_{21}\left(F_{1} A_{2}-D_{2} A_{1}\right)+c_{22}\left(H_{1} A_{2}-E_{2} A_{1}\right)}{B_{2} A_{1}-B_{1} A_{2}},
\end{gathered}
$$

where,

$$
\begin{aligned}
& A_{1}= \\
& \frac{-8 b^{2}\left(1-\varepsilon_{12}^{1}\right)\left(1-\varepsilon_{21}^{1}\right)+2\left(1-\varepsilon_{12}^{1}+\varepsilon_{21}^{1}\right)\left(1+\varepsilon_{12}^{1}-\varepsilon_{21}^{1}\right)}{\left(1-\varepsilon_{12}^{1}\right)\left((1+2 b)\left(1-\varepsilon_{21}^{1}\right)+\varepsilon_{12}^{1}\right)}, \\
& A_{3}= \\
& \frac{-8 b^{2}\left(1-\varepsilon_{12}^{1}\right)\left(1-\varepsilon_{21}^{1}\right)+2\left(1-\varepsilon_{12}^{1}+\varepsilon_{21}^{1}\right)\left(1+\varepsilon_{12}^{1}-\varepsilon_{21}^{1}\right)}{\left(1-\varepsilon_{21}^{1}\right)\left((1+2 b)\left(1-\varepsilon_{12}^{1}\right)+\varepsilon_{21}^{1}\right)}, \\
& B_{2}= \\
& \frac{-8 b^{2}\left(1-\varepsilon_{12}^{2}\right)\left(1-\varepsilon_{21}^{2}\right)+2\left(1-\varepsilon_{12}^{2}+\varepsilon_{21}^{2}\right)\left(1+\varepsilon_{12}^{2}-\varepsilon_{21}^{2}\right)}{\left(1-\varepsilon_{12}^{2}\right)\left((1+2 b)\left(1-\varepsilon_{21}^{2}\right)+\varepsilon_{12}^{2}\right)}, \\
& B_{4}= \\
& \frac{-8 b^{2}\left(1-\varepsilon_{12}^{2}\right)\left(1-\varepsilon_{21}^{2}\right)+2\left(1-\varepsilon_{12}^{2}+\varepsilon_{21}^{2}\right)\left(1+\varepsilon_{12}^{2}-\varepsilon_{21}^{2}\right)}{\left(1-\varepsilon_{21}^{2}\right)\left((1+2 b)\left(1-\varepsilon_{12}^{2}\right)+\varepsilon_{21}^{2}\right)}, \\
& A_{2}= \\
& \frac{4(1+2 b)\left(1-\varepsilon_{12}^{1}\right)\left(1-\varepsilon_{21}^{1}\right)+2\left(\varepsilon_{12}^{1}\left(1-\varepsilon_{12}^{1}\right)+\varepsilon_{21}^{1}\left(1-\varepsilon_{21}^{1}\right)\right)}{\left(1-\varepsilon_{12}^{1}\right)\left((1+2 b)\left(1-\varepsilon_{21}^{1}\right)+\varepsilon_{12}^{1}\right)}, \\
& A_{4}= \\
& \frac{4(1+2 b)\left(1-\varepsilon_{12}^{1}\right)\left(1-\varepsilon_{21}^{1}\right)+2\left(\varepsilon_{12}^{1}\left(1-\varepsilon_{12}^{1}\right)+\varepsilon_{21}^{1}\left(1-\varepsilon_{21}^{1}\right)\right)}{\left(1-\varepsilon_{21}^{1}\right)\left((1+2 b)\left(1-\varepsilon_{12}^{1}\right)+\varepsilon_{21}^{1}\right)}, \\
& \frac{4(1+2 b)\left(1-\varepsilon_{12}^{2}\right)\left(1-\varepsilon_{21}^{2}\right)+2\left(\varepsilon_{12}^{2}\left(1-\varepsilon_{12}^{2}\right)+\varepsilon_{21}^{2}\left(1-\varepsilon_{21}^{2}\right)\right)}{\left(1-\varepsilon_{12}^{2}\right)\left((1+2 b)\left(1-\varepsilon_{21}^{2}\right)+\varepsilon_{12}^{2}\right)}, \\
& B_{3}= \\
& \frac{4(1+2 b)\left(1-\varepsilon_{12}^{2}\right)\left(1-\varepsilon_{21}^{2}\right)+2\left(\varepsilon_{12}^{2}\left(1-\varepsilon_{12}^{2}\right)+\varepsilon_{21}^{2}\left(1-\varepsilon_{21}^{2}\right)\right)}{\left(1-\varepsilon_{21}^{2}\right)\left((1+2 b)\left(1-\varepsilon_{12}^{2}\right)+\varepsilon_{21}^{2}\right)}, \\
& D_{1}=\frac{2 b\left(1-\varepsilon_{12}^{1}-\varepsilon_{21}^{1}\right)}{(1+2 b)\left(1-\varepsilon_{21}^{1}\right)+\varepsilon_{12}^{1}}, \\
& D_{3}=\frac{4 b^{2}\left(1-\varepsilon_{12}^{1}\right)\left(1-\varepsilon_{21}^{1}\right)-2 \varepsilon_{12}^{1}\left(1+\varepsilon_{21}^{1}-\varepsilon_{12}^{1}\right)}{\left(1-\varepsilon_{21}^{1}\right)\left((1+2 b)\left(1-\varepsilon_{12}^{1}\right)+\varepsilon_{21}^{1}\right)}, \\
& D_{2}=\frac{2 b\left(1-\varepsilon_{12}^{2}-\varepsilon_{21}^{2}\right)}{(1+2 b)\left(1-\varepsilon_{21}^{2}\right)+\varepsilon_{12}^{2}}, \\
& D_{4}=\frac{4 b^{2}\left(1-\varepsilon_{12}^{2}\right)\left(1-\varepsilon_{21}^{2}\right)-2 \varepsilon_{12}^{2}\left(1+\varepsilon_{21}^{2}-\varepsilon_{12}^{2}\right)}{\left(1-\varepsilon_{21}^{2}\right)\left((1+2 b)\left(1-\varepsilon_{12}^{2}\right)+\varepsilon_{21}^{2}\right)}, \\
& E_{3}=\frac{2 b\left(1-\varepsilon_{12}^{1}-\varepsilon_{21}^{1}\right)}{(1+2 b)\left(1-\varepsilon_{12}^{1}\right)+\varepsilon_{21}^{1}}, \\
& E_{1}=\frac{4 b^{2}\left(1-\varepsilon_{12}^{1}\right)\left(1-\varepsilon_{21}^{1}\right)-2 \varepsilon_{21}^{1}\left(1+\varepsilon_{12}^{1}-\varepsilon_{21}^{1}\right)}{\left(1-\varepsilon_{12}^{1}\right)\left((1+2 b)\left(1-\varepsilon_{21}^{1}\right)+\varepsilon_{12}^{1}\right)},
\end{aligned}
$$$$
B_{1}=
$$ 


$$
\begin{aligned}
E_{4}= & \frac{2 b\left(1-\varepsilon_{12}^{2}-\varepsilon_{21}^{2}\right)}{(1+2 b)\left(1-\varepsilon_{12}^{2}\right)+\varepsilon_{21}^{2}}, \\
E_{2}= & \frac{4 b^{2}\left(1-\varepsilon_{12}^{2}\right)\left(1-\varepsilon_{21}^{2}\right)-2 \varepsilon_{21}^{2}\left(1+\varepsilon_{12}^{2}-\varepsilon_{21}^{2}\right)}{\left(1-\varepsilon_{12}^{2}\right)\left((1+2 b)\left(1-\varepsilon_{21}^{2}\right)+\varepsilon_{12}^{2}\right)}, \\
F_{1}= & \frac{2\left(\varepsilon_{12}^{2}+b\left(1-\varepsilon_{21}^{2}\right)\right)}{(1+2 b)\left(1-\varepsilon_{21}^{2}\right)+\varepsilon_{12}^{2}}, \\
F_{3}= & \frac{-2\left(1-\varepsilon_{12}^{2}\right)\left(\varepsilon_{12}^{2}+b\left(1-\varepsilon_{21}^{2}\right)\right)}{\left(1-\varepsilon_{21}^{2}\right)\left((1+2 b)\left(1-\varepsilon_{12}^{2}\right)+\varepsilon_{21}^{2}\right)}, \\
F_{2}= & \frac{2\left(\varepsilon_{12}^{1}+b\left(1-\varepsilon_{21}^{1}\right)\right)}{(1+2 b)\left(1-\varepsilon_{21}^{1}\right)+\varepsilon_{12}^{1}}, \\
F_{4}= & \frac{-2\left(1-\varepsilon_{12}^{1}\right)\left(\varepsilon_{12}^{1}+b\left(1-\varepsilon_{21}^{1}\right)\right)}{\left(1-\varepsilon_{21}^{1}\right)\left((1+2 b)\left(1-\varepsilon_{12}^{1}\right)+\varepsilon_{21}^{1}\right)}, \\
H_{3}= & \frac{2\left(\varepsilon_{21}^{2}+b\left(1-\varepsilon_{12}^{2}\right)\right)}{(1+2 b)\left(1-\varepsilon_{12}^{2}\right)+\varepsilon_{21}^{2}}, \\
H_{2}= & \frac{-2\left(1-\varepsilon_{21}^{1}\right)\left(\varepsilon_{21}^{1}+b\left(1-\varepsilon_{12}^{1}\right)\right)}{\left(1-\varepsilon_{12}^{1}\right)\left((1+2 b)\left(1-\varepsilon_{21}^{2}+b\left(1-\varepsilon_{12}^{2}\right)\right)\right.} \\
H_{1}= & \frac{2\left(\varepsilon_{21}^{1}+b\left(1-\varepsilon_{12}^{1}\right)\right)}{\left(1-\varepsilon_{12}^{2}\right)\left((1+2 b)\left(1-\varepsilon_{21}^{2}\right)+\varepsilon_{12}^{2}\right)}, \\
(1+2 b)\left(1-\varepsilon_{12}^{1}\right)+\varepsilon_{21}^{1} &
\end{aligned}
$$

The stability of this equilibrium solutions is based on the eigenvalues of the Jacobian matrix of the system (7), which is given by (10);

where

$$
\begin{aligned}
\gamma_{1}= & \left(1-\varepsilon_{12}^{1}\right)\left(a+b c_{11}-4 b p_{11}^{t}+p_{12}^{t}+p_{21}^{t}+p_{22}^{t}\right) \\
& +\varepsilon_{21}^{1}\left(p_{12}^{t}-c_{12}\right)
\end{aligned}
$$

and

$$
\begin{aligned}
\gamma_{2}= & \left(1-\varepsilon_{21}^{1}\right)\left(a+b c_{12}-4 b p_{12}^{t}+p_{11}^{t}+p_{21}^{t}+p_{22}^{t}\right) \\
& +\varepsilon_{12}^{1}\left(p_{11}^{t}-c_{11}\right)
\end{aligned} .
$$

The equilibrium solution will be stable if the eigenvalues $\lambda_{i}, i=1,2,3,4$ of the Jacobian matrix (10) satisfy the conditions $\left|\lambda_{i}\right|<1, i=1,2,3,4$.

The eigenvalues for the first equilibrium point $E_{1}$ are given by $\lambda_{1}=1+\alpha_{11} \gamma_{1}, \lambda_{2}=1+\alpha_{12} \gamma_{2}$, and

$$
\lambda_{3,4}= \pm \sqrt{\frac{\left(1-\varepsilon_{12}^{2}+\varepsilon_{21}^{2}\right)\left(1-\varepsilon_{21}^{2}+\varepsilon_{12}^{2}\right)}{4 b^{2}\left(1-\varepsilon_{12}^{2}\right)\left(1-\varepsilon_{21}^{2}\right)}} \text {. For the other }
$$

equilibrium points it is very difficult to compute these eigenvalues. Instead, we find the characteristic polynomial, which has the following form:

$$
P(\lambda)=\lambda^{4}+a_{1} \lambda^{3}+a_{2} \lambda^{2}+a_{3} \lambda+a_{4},
$$

Then, the necessary and sufficient conditions [17], for all roots of the characteristic polynomial $P(\lambda)$ to satisfy the conditions that $\left|\lambda_{i}\right|<1, i=1,2,3,4$ are the following:

$$
\begin{aligned}
& \text { 1) } P(1)=1+a_{1}+a_{2}+a_{3}+a_{4}>0, \\
& \text { 2) } P(-1)=1-a_{1}+a_{2}-a_{3}+a_{4}>0, \\
& \text { 3) }\left|a_{4}\right|<1, \\
& \text { 4) }\left|1-a_{4}^{2}\right|>\left|a_{3}-a_{4} a_{1}\right|, \\
& \text { 5) }\left|\left(1-a_{4}^{2}\right)^{2}-\left(a_{3}-a_{4} a_{1}\right)^{2}\right| \\
& >\left|a_{2}\left(1-a_{4}^{2}\right)^{2}-\left(a_{3}-a_{4} a_{1}\right)\left(a_{1}-a_{4} a_{3}\right)\right| .
\end{aligned}
$$

The coefficients of the characteristic polynomial for the most important one (coexistence) are as follows:

$$
\begin{aligned}
& a_{1}=-\left(2+\alpha_{11} \gamma_{1}+\alpha_{12} \gamma_{2}\right), \\
& a_{2}=\left(1+\alpha_{11} \gamma_{1}\right)\left(1+\alpha_{12} \gamma_{2}\right) \\
& -\alpha_{11} \alpha_{12} p_{11} p_{12}\left(1-\varepsilon_{12}^{1}+\varepsilon_{21}^{1}\right)\left(1-\varepsilon_{21}^{1}+\varepsilon_{12}^{1}\right) \\
& -\frac{\alpha_{11} p_{11}\left(1-\varepsilon_{12}^{1}\right)}{b}-\frac{\alpha_{12} p_{12}\left(1-\varepsilon_{21}^{1}\right)}{b} \\
& -\frac{\left(1-\varepsilon_{12}^{2}+\varepsilon_{21}^{2}\right)\left(1-\varepsilon_{21}^{2}+\varepsilon_{12}^{2}\right)}{4 b^{2}\left(1-\varepsilon_{12}^{2}\right)\left(1-\varepsilon_{21}^{2}\right)},
\end{aligned}
$$

$$
\left[\begin{array}{cccc}
1+\alpha_{11} \gamma_{1} & \alpha_{11} p_{11}\left(1-\varepsilon_{12}^{1}+\varepsilon_{21}^{1}\right) & \alpha_{11} p_{11}\left(1-\varepsilon_{12}^{1}\right) & \alpha_{11} p_{11}\left(1-\varepsilon_{12}^{1}\right) \\
\alpha_{12} p_{12}\left(1-\varepsilon_{21}^{1}+\varepsilon_{12}^{1}\right) & 1+\alpha_{12} \gamma_{2} & \alpha_{12} p_{12}\left(1-\varepsilon_{21}^{1}\right) & \alpha_{12} p_{12}\left(1-\varepsilon_{21}^{1}\right) \\
\frac{1}{2 b} & \frac{1}{2 b} & 0 & \frac{\left(1-\varepsilon_{12}^{2}+\varepsilon_{21}^{2}\right)}{2 b\left(1-\varepsilon_{12}^{2}\right)} \\
\frac{1}{2 b} & \frac{1}{2 b} & \frac{\left(1-\varepsilon_{21}^{2}+\varepsilon_{12}^{2}\right)}{2 b\left(1-\varepsilon_{21}^{2}\right)} & 0
\end{array}\right]
$$




$$
\begin{aligned}
a_{3}= & -\frac{\alpha_{11} p_{11}\left(1-\varepsilon_{12}^{1}\right)\left(\left(1-\varepsilon_{12}^{2}\right)\left(1-\varepsilon_{21}^{2}+\varepsilon_{12}^{2}\right)+\left(1-\varepsilon_{21}^{2}\right)\left(1-\varepsilon_{12}^{2}+\varepsilon_{21}^{2}\right)\right)}{4 b^{2}\left(1-\varepsilon_{12}^{2}\right)\left(1-\varepsilon_{21}^{2}\right)} \\
& -\frac{\alpha_{12} p_{12}\left(1-\varepsilon_{21}^{1}\right)\left(\left(1-\varepsilon_{12}^{2}\right)\left(1-\varepsilon_{21}^{2}+\varepsilon_{12}^{2}\right)+\left(1-\varepsilon_{21}^{2}\right)\left(1-\varepsilon_{12}^{2}+\varepsilon_{21}^{2}\right)\right)}{4 b^{2}\left(1-\varepsilon_{12}^{2}\right)\left(1-\varepsilon_{21}^{2}\right)} \\
& -\frac{\alpha_{11} \alpha_{12} p_{11} p_{12}\left(\left(1-\varepsilon_{12}^{1}\right)\left(1-\varepsilon_{21}^{1}+\varepsilon_{12}^{1}\right)+\left(1-\varepsilon_{21}^{1}\right)\left(1-\varepsilon_{12}^{1}+\varepsilon_{21}^{1}\right)\right)}{b} \\
& +\frac{\alpha_{11} p_{11}\left(1-\varepsilon_{12}^{1}\right)\left(1+\alpha 12 \gamma_{2}\right)+\alpha_{12} p_{12}\left(1-\varepsilon_{21}^{1}\right)\left(1+\alpha 11 \gamma_{1}\right)}{b}+\frac{\left(2+\alpha_{11} \gamma_{1}+\alpha_{12} \gamma_{2}\right)\left(1+\varepsilon_{12}^{2}-\varepsilon_{21}^{2}\right)\left(1-\varepsilon_{12}^{2}+\varepsilon_{21}^{2}\right)}{2 b^{2}\left(1-\varepsilon_{12}^{2}\right)\left(1-\varepsilon_{21}^{2}\right)}, \\
a_{4}= & \frac{\alpha_{11} \alpha_{12} p_{11} p_{12}\left(1-\varepsilon_{21}^{1}\right)\left(1-\varepsilon_{12}^{1}+\varepsilon_{21}^{1}\right)\left(\frac{1-\varepsilon_{21}^{2}+\varepsilon_{12}^{2}}{1-\varepsilon_{21}^{2}}+\frac{1-\varepsilon_{12}^{2}+\varepsilon_{21}^{2}}{1-\varepsilon_{12}^{2}}\right)}{4 b^{2}} \\
& -\frac{\alpha_{11} \alpha_{12} p_{11} p_{12}\left(1-\varepsilon_{12}^{1}\right)\left(1-\varepsilon_{21}^{1}+\varepsilon_{12}^{1}\right)}{4 b^{2}}\left(\frac{1-\varepsilon_{21}^{2}+\varepsilon_{12}^{2}}{1-\varepsilon_{21}^{2}}+\frac{1-\varepsilon_{12}^{2}+\varepsilon_{21}^{2}}{1-\varepsilon_{12}^{2}}\right) \\
+ & \frac{\alpha_{11} \alpha_{12} p_{11} p_{12}\left(1-\varepsilon_{21}^{1}+\varepsilon_{12}^{1}\right)\left(1-\varepsilon_{12}^{1}+\varepsilon_{21}^{1}\right)\left(1-\varepsilon_{21}^{2}+\varepsilon_{12}^{2}\right)\left(1-\varepsilon_{12}^{2}+\varepsilon_{21}^{2}\right)}{4 b^{2}\left(1-\varepsilon_{12}^{2}\right)\left(1-\varepsilon_{21}^{2}\right)} \\
+ & \frac{\alpha_{11} p_{11}\left(1-\varepsilon_{12}^{1}\right)\left(1+\alpha_{12} \gamma_{2}\right)\left(1-\varepsilon_{21}^{2}+\varepsilon_{12}^{2}+\frac{1-\varepsilon_{12}^{2}+\varepsilon_{21}^{2}}{1-\varepsilon_{12}^{2}}\right)+\frac{\alpha_{12} p_{12}\left(1-\varepsilon_{21}^{1}\right)\left(1+\alpha_{11} \gamma_{1}\right)}{4 b^{2}}\left(\frac{1-\varepsilon_{21}^{2}+\varepsilon_{12}^{2}}{1-\varepsilon_{21}^{2}}+\frac{1-\varepsilon_{12}^{2}+\varepsilon_{21}^{2}}{1-\varepsilon_{12}^{2}}\right)}{4 b^{2}} \\
& \left(1+\alpha_{11} \gamma_{1}\right)\left(1+\alpha_{12} \gamma_{2}\right)\left(1-\varepsilon_{21}^{2}+\varepsilon_{12}^{2}\right)\left(1-\varepsilon_{12}^{2}+\varepsilon_{21}^{2}\right) \\
&
\end{aligned}
$$

Then, the equilibrium solution $E_{4}$ of the system (7) is stable under the conditions (11). This means that in the long run all firms are coexist. So, the market will be stable.

\section{Numerical Simulations}

In this section, we will use some numerical simulations to show the complicated behaviour of the model (stability, period doubling bifurcation and chaos). Figure 1 shows the bifurcation diagram of the prices and profits with respect to the adjust speed $\alpha_{11}$ while the other parameters are constant and have taken the values $p_{11}^{0}=0.30, \quad p_{12}^{0}=0.55, \quad p_{21}^{0}=0.60, \quad p_{22}^{0}=0.64$, $\varepsilon_{12}^{1}=0.2, \quad \varepsilon_{21}^{1}=0.3, \varepsilon_{12}^{2}=0.1, \varepsilon_{21}^{2}=0.4, c_{11}=0.11$, $c_{12}=0.13, c_{21}=0.21, c_{22}=0.23, a=1, b=3$ and $\alpha_{12}=0.2$.

This figure shows that the equilibrium point $\bar{p}=(0.5212497,0.5254234,0.5638708,0.5599204)$ is locally stable for $\alpha_{11}<0.7569938$, after this value it became periodic and finally the system became chaotic. The same thing occur to the profits in figure 1B at the same value of $\alpha_{11}$.

Figure 2 shows the effect of changing the parameters $\varepsilon_{l j}^{i}$. We get a bifurcation diagram for the prices and profits with respect to $\varepsilon_{12}^{1}$ with the values of the other parameters are the same as in Figure 1 except that $\alpha_{11}$ became constant and takes the value $\alpha_{12}=0.9$ and $\varepsilon_{12}^{1}$ became variable.

We note that the small cooperation among the firms in the same team $\left(\varepsilon_{12}^{1}<0.3459991\right)$ will lead to a complex behaviour in the system, while the increasing this cooperation will lead to the stability.

\section{Chaos Control}

As we seen in the last section, the adjustment rate $\alpha_{i j}$ and the payoff return $\varepsilon_{l j}^{i}$ of the boundedly rational firms play an important role in the stability of the market. So, to avoid this complexity we will try to control the chaos. We will use the feedback method [18] to control the adjustment magnitude. Modifying the first equation in our system will give us the following controlled system; 

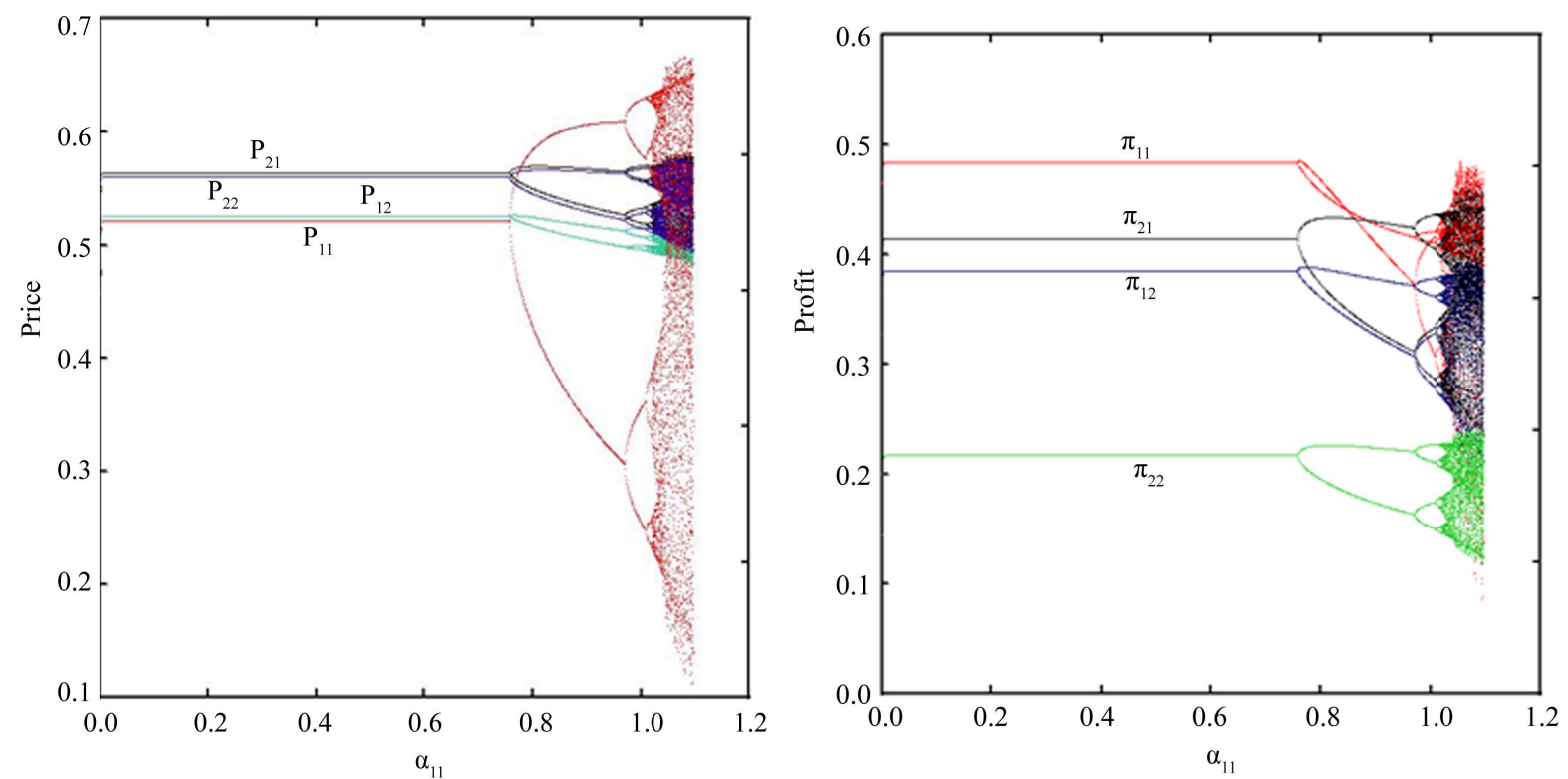

Figure 1. The bifurcation diagram of the prices and the profits with respect to $\alpha_{11}$.
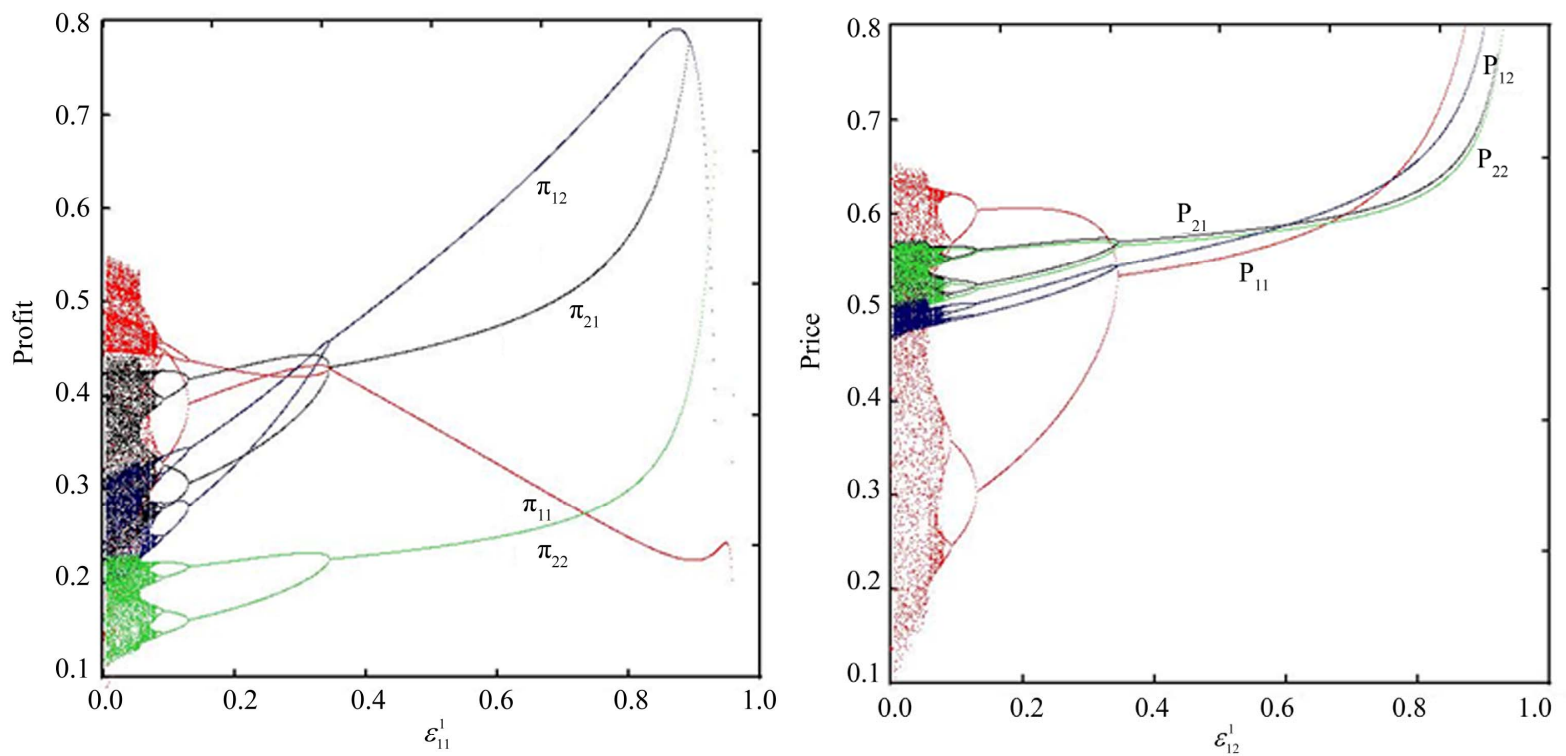

Figure 2. The bifurcation diagram of the prices and the profits with respect to $\varepsilon_{12}^{1}$.

$$
\begin{aligned}
& p_{11}^{t+1}=p_{11}^{t}+\frac{\alpha_{11}}{k+1} p_{11}^{t}\left[\left(1-\varepsilon_{12}^{1}\right)\left(a+b c_{11}-2 b p_{11}^{t}+p_{12}^{t}+p_{21}^{t}+p_{22}^{t}\right)+\varepsilon_{21}^{1}\left(p_{12}^{t}-c_{12}\right)\right] \\
& p_{12}^{t+1}=p_{12}^{t}+\alpha_{12} p_{12}^{t}\left[\left(1-\varepsilon_{21}^{1}\right)\left(a+b c_{12}-2 b p_{12}^{t}+p_{11}^{t}+p_{21}^{t}+p_{22}^{t}\right)+\varepsilon_{12}^{1}\left(p_{11}^{t}-c_{11}\right)\right] \\
& p_{21}^{t+1}=\frac{1}{2 b}\left(a+b c_{21}+p_{22}^{t}+p_{11}^{t}+p_{12}^{t}\right)+\frac{\varepsilon_{21}^{2}}{2 b\left(1-\varepsilon_{12}^{2}\right)}\left(p_{22}^{t}-c_{22}\right) \\
& p_{22}^{t+1}=\frac{1}{2 b}\left(a+b c_{22}+p_{21}^{t}+p_{11}^{t}+p_{12}^{t}\right)+\frac{\varepsilon_{12}^{2}}{2 b\left(1-\varepsilon_{21}^{2}\right)}\left(p_{21}^{t}-c_{21}\right)
\end{aligned}
$$

where the parameter $k>0$ is the control factor. The Jacobian matrix of the controlled system will be: 


$$
\left[\begin{array}{cccc}
1+\frac{\alpha_{11} \gamma_{1}}{k+1} & \frac{\alpha_{11} p_{11}\left(1-\varepsilon_{12}^{1}+\varepsilon_{21}^{1}\right)}{k+1} & \frac{\alpha_{11} p_{11}\left(1-\varepsilon_{12}^{1}\right)}{k+1} & \frac{\alpha_{11} p_{11}\left(1-\varepsilon_{12}^{1}\right)}{k+1} \\
\alpha_{12} p_{12}\left(1-\varepsilon_{21}^{1}+\varepsilon_{12}^{1}\right) & 1+\alpha_{12} \gamma_{2} & \alpha_{12} p_{12}\left(1-\varepsilon_{21}^{1}\right) & \alpha_{12} p_{12}\left(1-\varepsilon_{21}^{1}\right) \\
\frac{1}{2 b} & \frac{1}{2 b} & 0 & \frac{\left(1-\varepsilon_{12}^{2}+\varepsilon_{21}^{2}\right)}{2 b\left(1-\varepsilon_{12}^{2}\right)} \\
\frac{1}{2 b} & \frac{1}{2 b} & \frac{\left(1-\varepsilon_{21}^{2}+\varepsilon_{12}^{2}\right)}{2 b\left(1-\varepsilon_{21}^{2}\right)} & 0
\end{array}\right]
$$

The original system is chaotic for the parameter values $p_{11}^{0}=0.30, \quad p_{12}^{0}=0.55, \quad p_{21}^{0}=0.60, \quad p_{22}^{0}=0.64$, $\varepsilon_{12}^{1}=0.2, \varepsilon_{21}^{1}=0.3, \varepsilon_{12}^{2}=0.1, \varepsilon_{21}^{2}=0.4, c_{11}=0.11$, $c_{12}=0.13, \quad c_{21}=0.21, \quad c_{22}=0.23, a=1, b=3$, $\alpha_{11}=1.1$ and $\alpha_{12}=0.2$. But the controlled system is stable $\left(\lambda_{i}<1, i=1,2,3,4\right)$ for all the above parameters values and for $k>0.4559977$.

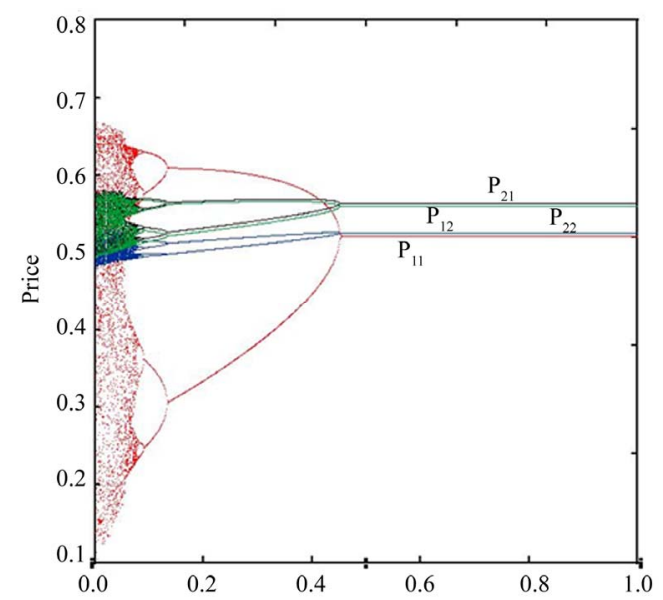

Figure 3. The bifurcation diagram of the prices with respect to the controlling factor $k$.

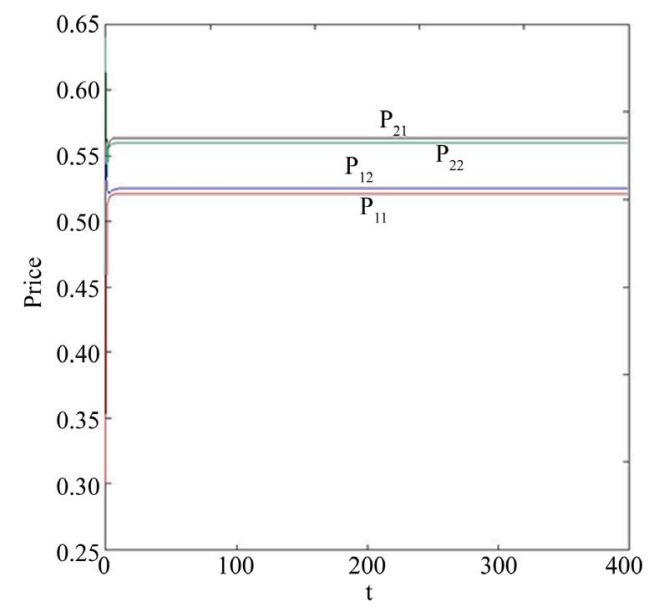

Figure 4. The bifurcation diagram of the prices with respect to the controlling factor $\boldsymbol{k}=\mathbf{0 . 5}$.
From Figure 3, we find that the controlled system begin chaotic, periodic and then stable by increasing the control factor $k$.

Figure 4 shows the stability behaviour of the controlled system when $k=0.5$. This means that if the firms in the first team adopt the feedback adjustment, the price system can switch from a chaotic to a regular or equilibrium state.

\section{Acknowledgements}

We would like to express our appreciation to the Deanship of Scientific Research at King AbdulAziz University, Saudi Arabia for its financial support of this study, Grant No. 3-058/430.

\section{References}

[1] R. Gibbons, "A Primer in Game Theory," Simon and Schuster, New York, 1992.

[2] B. R. Myerson, "Game Theory: Analysis of Conflict," Harvard University Press, Cambridge, 1997.

[3] A. Cournot, "Researches into the Mathematical Principles of the Theory of Wealth," Macmillan, New York, 1897.

[4] J. Bertrand, "Theorie Mathematique de la Richesse Soaiale," Journal des Savants, Vol. 67, 1883, pp. 499508.

[5] J. Nash, "Equilibrium Points in an n-Person Games," Proceedings of the National Academy of Sciences, Vol. 36, No. 1, 1950, pp. 48-49. doi:10.1073/pnas.36.1.48

[6] E. Ahmed, A. S. Hegazi, M. F. Elettreby and S. S. Askar, "On Multi-Team Games," Physica A: Statistical Mechanics and Its Applications, Vol. 369, No. 2, 2006, pp. 809816. doi:10.1016/j.physa.2006.02.011

[7] T. Puu, "Chaos in Duopoly Pricing," Chaos, Solitons \& Fractals, Vol. 1, No. 6, 1991, pp 573-581.

[8] T. Puu, "The Chaotic Monopolist," Chaos, Solitons \& Fractals, Vol. 5, No. 1, 1995, pp. 35-44.

[9] M. F. Elettreby and S. Z. Hassan, "Dynamical MultiTeam Cournot Game," Chaos, Solitons \& Fractals, Vol. 27, No. 3, 2006, pp. 666-672.

[10] E. Ahmed and A. S. Hegazi, On Dynamical Multi-Team 
and Signaling Games," Applied Mathematics and Computation, Vol. 172, No. 1, 2006, pp. 524-530. doi:10.1016/j.amc.2005.02.030

[11] S. S. Asker, "On Dynamical Multi-Team Cournot Game in Exploitation of a Renewable Resource," Chaos, Solitons \& Fractals, Vol. 32, No. 1, 2007, pp. 264-268.

[12] E. Ahmed, M. F. Elettreby and A. S. Hegazi, "On Puu's Incomplete Information Formulation for the Standard and Multi-Team Bertrand Game," Chaos, Solitons \& Fractals, Vol. 30, No. 5, December 2006, pp. 1180-1184.

[13] J. X. Zhang, Q. L. Da and Y. H. Wang, "The Dynamics of Bertrand Model with Bounded Rationality," Chaos, Solitons \& Fractals, Vol. 39, No. 5, 2009, pp. 20482055.

[14] D. Zhanwen, H. Qinglan and Y. Honglin, “Analysis of the Dynamics of Multi-Team Bertrand Game with Heterogeneous Players," International Journal of Systems Science, Vol. 42, No. 6, 2010, pp. 1047-1056.

[15] G. Gigerenzer and R. Selten, "Bounded Rationality," MIT Press, Cambridge, 2002.

[16] L. Edelstein-Keshet, "Mathematical Models in Biology," Random House, New York, 1988.

[17] E. I. Jury, "The Inners Approch to Some Problems of System Theory," IEEE Transactions on Automatic Control, Vol. 16, No. 3, 1971, pp. 233-241. doi:10.1109/TAC.1971.1099725

[18] E. M. Elabbasy, H. N. Agiza and A. A. Elsadany, "Analysis of Nonlinear Triopoly Game with Heterogeneous Players," Computers \& Mathematics with Applications, Vol. 57, No. 3, 2009, pp. 488-499. 